\title{
MIGRACIONES CAUSADAS POR LA SUBIDA DEL NIVEL DEL MAR: UN RETO PARA EL DERECHO INTERNACIONAL
}

\section{Migrations Caused by Sea LeVel Rise: A Challenge for International LAW}

\author{
ESTELA MARTín PASCUAL \\ Investigadora predoctoral en formación \\ Contrato cofinanciado por la Junta de Castilla y León y el Fondo Social Europeo \\ Universidad de Salamanca \\ estelitamp@usal.es
}

Recibido: 30 de marzo de 2018 / Aceptado: 3 de septiembre de 2018

RESUMEN: Este trabajo analiza cómo la subida del nivel del mar, consecuencia del cambio climático, tiene una serie de implicaciones tanto para los Estados como para su población, siendo la más grave de ellas la desaparición del territorio de los Estados insulares de baja altitud. Dichas consecuencias darían lugar a movimientos migratorios tanto internos como internacionales. Sin embargo, se pone de manifiesto que estos migrantes climáticos no reciben ningún tipo de protección internacional, ya que, además de que no se les puede considerar refugiados, la comunidad internacional no ha creído oportuno, hasta el momento, adoptar ningún instrumento jurídicamente vinculante que los ampare y les otorgue una serie de derechos.

RESUM: Aquest treball analitza com la pujada de nivell del mar, conseqüència del canvi climàtic, manté una sèrie d'implicacions tant per als estats com per a la seva població, el més greu dels quals és la desaparició del territori dels estats insulars de baixa altitud. Aquestes conseqüències donarien lloc a moviments migratoris tant interns com internacionals. Malgrat tot, es posa de manifest que aquests migrants climàtics no reben cap tipus de protecció internacional, ja que, a més que no se'ls pot considerar refugiats, la comunitat internacional no ha cregut oportú fins ara d'adoptar 
cap instrument jurídicament vinculant que els empari i que els atorgui una sèrie de drets.

ABSTRACT: This article examines how the sea-level rise, a consequence of climate change, has several implications, both for the States and their population. The most serious of these would be the disappearance of the territory of the low-lying island states. Those consequences would result in internal and international population movements. However, it is revealed that these climate migrants do not receive any kind of international protection, because apart from the fact that they cannot be considered as refugees, the international community has not been willing to adopt any legally binding instrument so far to protect and granting them a range of rights.

PALABRAS CLAVE: cambio climático, subida del nivel del mar, territorio, migrantes climáticos, refugiados climáticos

PARAULES CLAU: canvi climàtic, pujada del nivell del mar, territori, migrants climàtics, refugiats climàtics

KEY WORDS: climate change, sea-level rise, territory, climate migrants, climate refugees

SUMARIO: I. Introducción. II. Retos. 1. La potencial desaparición del territorio de un Estado y el problema de la apatridia. 2. La protección jurídica que reciben los migrantes por causas medioambientales y la problemática existente. III. Posibles soluciones. 1. Instrumentos existentes. 2. Nuevos instrumentos. IV. Conclusiones. V. Bibliografía.

\section{INTRODUCCIÓN}

Si bien es cierto que, como apunta Díez de Velasco ${ }^{1}$, no contamos con una definición de Estado en ningún texto jurídico internacional, se ha venido entendiendo que estos sujetos internacionales "nacen" cuando consiguen reunir los siguientes tres elementos: territorio, población y organización política, siguiendo la Convención de

1 DÍEZ DE VELASCO, M., Instituciones de derecho internacional público, Ed. Tecnos, Madrid, 2009, $17^{\mathrm{a}}$ ed., p. 275. 
Montevideo de $1933^{2}$ y la más reciente Comisión Badinter ${ }^{3}$. Y es en el momento en el que falla alguno de ellos cuando nos encontramos ante un Estado fallido o en descomposición.

El cambio climático tiene consecuencias también en este campo, proyectando un escenario hasta hace muchos años impensable, ya que plantea la posibilidad de que un Estado devenga inhabitable a causa de las continuas inundaciones por la subida del nivel del mar y, finalmente, desaparezca.

Por tanto, a las amenazas clásicas que desestabilizan y ponen en peligro los tres elementos del Estado, como rebeliones internas, guerras civiles o conflictos armados, se viene a sumar la subida del nivel del mar ligada al cambio climático, que podría poner en jaque al Estado al acabar con su elemento territorio y dejar, a su vez, a toda una población en situación de apatridia. Las Naciones Unidas ya dieron cuenta de esta amenaza en $2009^{4}$.

Planteado el peor de los escenarios posibles, es decir, la desaparición del elemento territorio de un Estado (o, si se prefiere, su sumersión bajo las aguas del océano), la subida del nivel del mar a causa del cambio climático también puede provocar inicialmente desplazamientos de población por las condiciones meteorológicas extremas o las inundaciones previas a la desaparición del territorio, que dificultan o hacen imposible el acceso al agua potable o el cultivo del suelo, a la vez que suponen amenazas potenciales tanto para los seres humanos como para determinados recursos productivos y económicos.

Sin embargo, y pese a que las consecuencias del cambio climático ya son tangibles, el derecho internacional va un paso por detrás, pues no hay un reconocimiento del

\footnotetext{
2 El artículo primero de la Convención sobre los Derechos y Deberes de los Estados de Montevideo de 1933 (disponible en: http://www.oas.org/juridico/spanish/tratados/a-40.html) contempla, además, un cuarto elemento: la capacidad del Estado de entablar relaciones internacionales con otros Estados, algo que autores como J. Crawford o Remiro Brotons y la propia Comisión Badinter engloban dentro de la nota característica de la soberanía, que vendría a ser una consecuencia de la estatalidad más que un cuarto elemento.

${ }^{3}$ Informe número 1 Comisión de Arbitraje para Yugoslavia, de 29 de noviembre de 1991, sobre la sucesión de las repúblicas de la República Federativa Socialista de Yugoslavia, Casos y Textos, núm. 62, p. 237.

4 "Muchos Estados insulares se enfrentan a la perspectiva de perder extensiones importantes de su territorio debido al aumento del nivel del mar y a las inundaciones, y algunos podrían quedar completamente bajo las aguas, con la amenaza consiguiente de apatridia para sus poblaciones". "EI cambio climático y sus posibles repercusiones para la seguridad", Documento A/64/350, p. 24, disponible en: http://www.acnur.org/t3/fileadmin/Documentos/BDL/2009/7257.pdf?view=1.
} 
estatus de refugiado para las personas que tienen que desplazarse, cruzando sus fronteras, por causas medioambientales, ni se les reconoce como categoría merecedora de protección en ningún texto legalmente vinculante a escala internacional.

Por el contrario, sí que existen algunos instrumentos jurídicos que recogen y dotan de protección la figura del desplazado interno. Como soft law y, por ende, no vinculantes, destacan los Principios Rectores de los desplazamientos internos de Naciones Unidas de 1998, reconocidos como un importante marco internacional de protección de estas personas. La Convención de Kampala de 2012, por su parte, recogió dichos principios en su articulado, de forma que los convirtió en vinculantes en su ámbito regional de aplicación territorial ${ }^{5}$.

Ante la laguna jurídica en torno a los migrantes climáticos internacionales, son varias las vías de actuación que se vienen planteando, tales como modificar la Convención de Ginebra para ampliar la definición de refugiado, elaborar un tratado internacional específico para las personas que se ven obligadas a abandonar su territorio por causas medioambientales o abordar estas situaciones mediante tratados bilaterales, entre otras.

\section{RETOS}

No se pueden ignorar las implicaciones que el cambio climático tiene para los Estados y sus poblaciones, materializadas por lo que concierne a este estudio en una subida del nivel del mar. Si las previsiones de los expertos se cumplen, dentro de varios años podríamos asistir a la desaparición del territorio de los pequeños Estados insulares de baja altitud, lo que dejaría, en principio, en situación de apatridia a sus ciudadanos.

Conscientes de la realidad que ya afrontan en forma de inundaciones constantes en las zonas costeras más bajas, con todo lo que ello conlleva para el suelo cultivable, la economía, las infraestructuras o el acceso al agua potable, y, más aún, conscientes también de que lo peor está por llegar, los Gobiernos de estos países han lanzado

\footnotetext{
5 JAKSA, B. y SMITH, J., "África: de los principios voluntarios a las normas vinculantes", Revista Migraciones Forzadas, núm. especial, diciembre 2008, pp.18 y 19.
} 
una llamada de auxilio a la comunidad internacional ${ }^{6}$. Solicitan un esfuerzo por avanzar en la dirección de proteger sobre todo a la población afectada por estos impactos climáticos, que se ve en una situación de desprotección jurídica internacional muy preocupante. A su vez, dichos Gobiernos están llevando a cabo, desde hace años y en la medida de sus posibilidades, diferentes iniciativas para lidiar con los efectos que está teniendo en su territorio la subida del nivel del mar, así como para adelantarse a los escenarios más desoladores que se avecinan.

\section{La potencial desaparición del territorio de un Estado y el problema de la apatridia}

Los datos obtenidos tanto por el Grupo Intergubernamental de Expertos sobre el Cambio Climático (IPCC) como por otros estudios relativos a la subida del nivel del mar para los próximos años muestran que determinados Estados están abocados a enfrentarse al peor de los escenarios posibles: la desaparición de su elemento territorio.

El quinto informe de evaluación de los expertos del IPCC prevé una elevación media del nivel del mar de entre 25 y 30 centímetros para 2065, y de 40 a 63 centímetros para 2100, dependiendo del escenario de emisión ${ }^{7}$ en el que nos movamos, pudiendo darse escenarios de mitigación, estabilización o de niveles muy altos de emisiones de gases de efecto invernadero.

Uno de los análisis más recientes, que acaba de publicarse en febrero de este año, apunta en la misma dirección que el panel de expertos, pues señala que, si los océanos continúan incrementando su nivel al ritmo actual, el nivel del mar podrá aumentar hasta 65 centímetros para $2100^{8}$.

\footnotetext{
${ }^{6}$ Una de las iniciativas más llamativas en este sentido fue la reunión que el gabinete del Gobierno de las Maldivas mantuvo en 2009 en el fondo marino, donde se firmó un documento que reclamaba la reducción de emisiones de carbono. Vid. http://news.bbc.co.uk/2/hi/8311838.stm.

${ }^{7}$ Estos escenarios son las denominadas trayectorias de concentración representativas. Hablamos de vías o trayectorias porque permiten obtener proyecciones de las concentraciones de GEI siguiendo su trayectoria a través del tiempo.

${ }^{8}$ NEREM, R. S., BECKLEY, B. D., FASULLO, J. T., HAMLINGTON, B. D., MASTERS, D. y MITCHUM, G. T., "Climate-change-driven accelerated sea-level rise", Proceedings of the National Academy of Sciences, 2018, disponible

en: http://www.pnas.org/content/pnas/early/2018/02/06/1717312115.full.pdf.
} 
Sin embargo, otras fuentes prevén aumentos incluso mayores. Por ejemplo, el estudio llevado a cabo por los climatólogos Robert DeConto y David Pollard sugiere que las estimaciones más recientes del IPCC para el futuro aumento del nivel del mar durante los próximos 100 años podrían ser demasiado bajas, y que esta subida podría casi doblarlas 9 .

En la misma línea se pronuncia otro trabajo de 2017 en el que participó la Universidad Complutense de Madrid, que señala que el nivel del mar podría aumentar entre los 30 y los 130 centímetros para 2100 en un escenario con altas emisiones de GEI ${ }^{10}$.

El mencionado informe del IPCC también recoge las principales causas de la elevación del nivel del mar. La primera de ellas es la expansión o dilatación térmica de los océanos (esto es, que el agua se expande en la medida en que se calienta). Otra causa importante es la suma de grandes cantidades de agua provenientes de los glaciares y zonas heladas derretidos debido al calentamiento global, como ya está sucediendo, por ejemplo, con el Ártico y la Antártida. En los polos el deshielo deja superficies más oscuras en el medio marino y en el terrestre, lo que, a su vez, acelera el procedimiento al absorber la luz solar en lugar de reflejarla y da lugar a una especie de círculo vicioso o espiral diabólica.

Pese a que estos datos son muy preocupantes, no lo son del mismo modo para todos los países. Si se confirmasen, la mayoría de Estados ribereños vería retroceder en mayor o menor medida sus líneas de costa, mientras que los pequeños Estados insulares de baja altitud, como Tuvalu, Kiribati, las Maldivas o las Islas Marshall, que se encuentran a una media de dos metros sobre el nivel del mar ${ }^{11}$, podrían sufrir el hundimiento total de su territorio, con todo lo que ello implicaría tanto para su población como para la propia condición de Estado.

\footnotetext{
9 DECONTO, R. y POLLARD, D., "Contribution of Antarctica to past and future sea-level rise", Nature, no. 531, 2016, pp. 591-597.

10 MENGEL, M., LEVERMANN, A., FRIELER, K., ROBINSON, A., MARZEION, B. y WINKELMANN, R., "Future sea level rise constrained by observations and long-term commitment", Proceedings of the National Academy of Sciences, vol. 113, núm. 10, 2016, pp. 2597-2602, disponible en: http://www.pnas.org/content/pnas/113/10/2597.full.pdf.

11 Tuvalu's National Adaptation Program of Action, 2007, p. 13, disponible en: http://unfccc.int/resource/docs/napa/tuv01.pdf; National Adaptation Program of Action, Republic of Maldives, 2007, pp. 20 y 77, disponible en: https://unfccc.int/resource/docs/napa/mdv01.pdf; National Adaptation Program of Action, 2007, Republic of Kiribati, 2007, p. 3; https://www.nytimes.com/interactive/2015/12/02/world/The-Marshall-Islands-Are-Disappearing.html.
} 
En este escenario, pues, de desaparición de uno de los elementos que le confieren la estatalidad a un Estado, surge la pregunta de si este desaparecería y las consecuencias que ello tendría para sus habitantes.

En principio, si pensamos en el derecho internacional clásico, la respuesta sería afirmativa. Sin embargo, la práctica nos demuestra que los Estados se resisten a declarar la desaparición de un Estado establecido aunque reúna las condiciones para ser calificado de fallido, acudiéndose habitualmente al principio de continuidad ${ }^{12}$. Según este, las alteraciones que puedan producirse en la organización política interna de un Estado no afectan, en principio, a la condición internacional de este, salvo en el caso de la desaparición de todo Gobierno. Ello quiere decir que, con base en el principio de la seguridad jurídica que debe regir las relaciones internacionales, cualesquiera cambios sobrevenidos en el régimen político de un Estado dejan inalteradas sus obligaciones internacionales frente a terceros países.

Sin embargo, los únicos precedentes hacen referencia al elemento Gobierno cuando este fracasa en su cometido de controlar su territorio y su población. En este sentido, tenemos el ejemplo claro de Somalia, Estado en el que falla el elemento Gobierno, en manos de Naciones Unidas, y cuyo presupuesto de controlar tanto el territorio como a la población queda reducido a una mínima parte del centro de su capital, Mogadiscio. En estos casos, lo habitual es acudir al principio de continuidad y, si hablamos de extinción de un Estado, se vincula con la sucesión de Estados ${ }^{13}$, principio según el cual un nuevo Estado reemplaza al extinto en la responsabilidad de las relaciones internacionales ${ }^{14}$.

Pero la pérdida del elemento territorio a causa de la subida del nivel del mar, o incluso el desplazamiento de la totalidad de la población junto con el Gobierno mismo al territorio de otro Estado, es un nuevo horizonte sin precedentes.

\footnotetext{
12 BORRÁS PENTINAT, S., "La migración ambiental: entre el abandono, el refugio y la protección internacional", PAPELES de relaciones ecosociales y cambio global, núm. 132, 2016, pp. 39-40; MCADAM, J., "El desplazamiento provocado por el cambio climático y el derecho internacional", Evento paralelo al Diálogo del Alto Comisionado sobre los desafíos en materia de protección, Ginebra, 2010, p. 7, disponible en: http://www.acnur.org/fileadmin/Documentos/BDL/2011/7460.pdf?view=1.

${ }^{13}$ PARK, S., "El cambio climático y el riesgo de apatridia: la situación de los Estados insulares bajos", Series de investigación: política de protección y asesoría legal, División de Protección Internacional (ACNUR), Ginebra, 2011, p. 13, disponible en: http://www.acnur.org/fileadmin/Documentos/BDL/2013/9056.pdf.

${ }^{14}$ Artículo 2.1 de la Convención de Viena sobre la sucesión de Estados en materia de bienes, archivos y deudas del Estado, de 1983.
} 
En este contexto habría que establecer las posibilidades de actuación planificada que se le presentan a ese Estado para proteger a su población: una migración masiva o una evacuación por una situación de emergencia, tesituras ambas indeseables y que deben evitarse en la medida de lo posible.

Por lo tanto, se pueden dar dos situaciones consecutivas en el tiempo. En la primera, antes de quedar sumergido de forma permanente por el océano, el territorio devendría inhabitable a causa de las continuas inundaciones, la erosión del terreno y la salinización de los acuíferos y de los cultivos. En este caso, dichas condiciones desfavorables podrían afectar exclusivamente a una parte del territorio, por lo que habría que aplicar estrategias de adaptación consistentes en la reubicación de la población de esas zonas a otra parte del Estado, normalmente hacia el interior ${ }^{15}$. En estas situaciones sería muy deseable seguir las recomendaciones fijadas por los Principios Rectores de los desplazamientos internos, que establecen que dicho traslado se llevará a cabo de forma que no menoscabe los derechos fundamentales a la vida, la dignidad, la libertad y la seguridad de los afectados ${ }^{16}$, garantizando al mismo tiempo unas condiciones satisfactorias de seguridad, alimentación, salud e higiene y sin separar a los miembros de una misma familia ${ }^{17}$.

Además de las medidas de reubicación, otra opción sería negociar acuerdos sobre migración con otros Estados, probablemente vecinos, que permitan un flujo ordenado y gradual de personas afectadas por estas circunstancias. Cabría la posibilidad de partir de iniciativas que ya se están llevando a cabo, si bien con otra finalidad, como la Categoría de Acceso del Pacífico, adaptándolas a las nuevas circunstancias.

$\mathrm{Si}$, por el contrario, la totalidad del territorio del Estado deviniera inhabitable para el ser humano, las consecuencias serían las mismas que en la segunda de las situaciones expuestas. En este caso, el Estado perdería su elemento territorio, ante lo cual tendría varias opciones.

Una de ellas, ya puesta en práctica por algunos países, sería la compra de tierras en otro Estado para trasladar allí a su población en caso necesario. Sin embargo, aunque esta transacción privada entre dos sujetos soberanos conlleva la propiedad sobre una

\footnotetext{
${ }^{15}$ PARK, "El cambio climático...", cit., p. 32.

16 Principio número 8.

17 Principio número 7.
} 
extensión de terreno determinada, no otorga soberanía al país adquirente ni da lugar a la creación de un nuevo Estado ${ }^{18}$. Por lo tanto, el Gobierno del país que se traslada (si se sigue reconociendo como tal aunque ya no exista su elemento territorial) tendría serias dificultades prácticas a la hora de ejercer sus funciones, ya que su ámbito de acción dependería de los derechos que el Estado donde se localiza el territorio decidiera otorgarle.

Esbozada esta alternativa y antes de pasar a analizar lo que ocurriría con la población que se trasladaría a ese nuevo lugar, conviene detenerse para analizar el concepto de apatridia y plantearse si es lo mismo no tener Estado que ser apátrida.

En primer lugar, se debe señalar que, aunque la Declaración Universal de los Derechos Humanos recoge, en su artículo 15, el derecho de toda persona a tener una nacionalidad, no existe para los Estados una correlativa obligación de otorgarla, sino que cada país es soberano a la hora de establecer los requisitos de adquisición y pérdida de tal condición.

A los efectos del artículo 1 de la Convención sobre el Estatuto de los Apátridas de 1954, fruto de la situación en la que las dos guerras mundiales habían dejado a un ingente número de personas, por apátrida se entiende "toda persona que no sea considerada como nacional suyo por ningún Estado, conforme a su legislación". Esa sería la definición, por tanto, de apátrida de iure.

Se debe tener presente que esta convención se elaboró en un período determinado para unos objetivos concretos, al igual que ocurrió con la Convención de Ginebra, por lo que no es de extrañar que no se contemplara explícitamente en aquella la pérdida de nacionalidad de los ciudadanos de un Estado por la desaparición física de este. Sin embargo, una lectura del texto puede llevarnos a interpretar que, si el Estado del que es nacional una persona pierde la condición de tal al desaparecer, la vinculación necesaria entre el Estado y la persona que da lugar a la nacionalidad no subsistiría, por lo que, a nuestro modo de ver, las personas de ese país entrarían en la definición del artículo 1. En definitiva, si un Estado deja de existir, difícilmente podrá reconocer

18 YASUNAGA KUMANO, M., "Los Pequeños Estados Insulares en Desarrollo y los desplazados climáticos", IEEE, núm. 4, 2016, p. 508. 
a nadie como nacional suyo, ofrecerle algún tipo de protección o garantizar sus derechos.

Pero incluso si ponemos en duda que esto sea así, nos restaría aún acudir al término "apátrida de facto", que sería aquella persona que se encuentra fuera del país de su nacionalidad y que no puede o, por razones válidas, no está dispuesta a acogerse a la protección de ese país ${ }^{19}$. Se podría defender que la situación de las personas a las que nos estamos refiriendo encajaría también en el primero de los supuestos, pues no se puede disponer de la protección de un país que ya no ostenta la calidad de tal y, aunque dicha condición de Estado subsistiese, difícilmente un Gobierno en el exilio permanente podría desplegar protección alguna sobre una población también "expulsada" de un territorio inhabitable. No obstante, la inclusión de los nacionales de los Estados insulares de baja altitud en este supuesto y no bajo el paraguas de la apatridia de iure los privaría de la mayor protección que esta última contempla (obviando el hecho del escaso número de Estados que han ratificado la Convención). Esto es así ya que el concepto de apátrida de facto no está contemplado en ningún texto internacional jurídicamente vinculante, pese a que sí se alzaron voces favorables a extender la definición de apátrida del artículo 1 del Convenio de 1954 a las situaciones de apatridia de facto $^{20}$. Finalmente, se adoptó una recomendación que contemplaba estos casos a través del Acta Final de la Convención sobre el Estatuto de los Apátridas, que carece de obligatoriedad jurídica: "The Conference recommends that persons who are stateless de facto should as far as possible be treated as stateless de jure to enable them to acquire an effective nationality"21.

En cualquier caso, la diferencia entre ambos términos no está en el desenlace o las consecuencias de ostentar una condición u otra, sino que, como señala Robinson ${ }^{22}$, en el supuesto de la apatridia de iure, el elemento clave es la ausencia de nacionalidad. Por el contrario, la idea de la apatridia de facto tiene un sentido más

\footnotetext{
${ }^{19}$ Reunión de Expertos organizada por la Oficina del Alto Comisionado de las Naciones Unidas para los Refugiados, "El Concepto de Personas Apátridas bajo el Derecho Internacional", Italia, mayo 2010, p. 5, disponible en: http://www.refworld.org/cgibin/texis/vtx/rwmain/opendocpdf.pdf?reldoc=y\&docid=4cea266d2.

20 VAN WAAS, L., Nationality Matters. Statelessness under international law, Intersentia, Amberes, 2008, p. 21.

${ }^{21}$ Artículo 1 del Acta Final de la Convención sobre el Estatuto de los Apátridas, disponible en: http://www.refworld.org/pdfid/3ae6b39620.pdf.

${ }_{22}$ FRIPP, E., Nationality and statelessness in the international law of refugee status, Bloombsbury, Londres, 2016, p. 97.
} 
amplio, pues el individuo, aunque no se vea privado de su nacionalidad, sí carece de los derechos vinculados a ella, fundamentalmente la protección diplomática y la entrada o el regreso al país de su nacionalidad.

Para ilustrar esta idea de que estas poblaciones quedarían en una situación de apatridia, tomemos como referencia el caso Nottebohm, en cuya sentencia se expone lo siguiente: "La nacionalidad es un vínculo jurídico que tiene como base un hecho social de arraigamiento, una solidaridad efectiva de existencia de intereses, junto con la existencia de derechos y deberes recíprocos. [...] Otorgada por un Estado, únicamente le faculta a él para ejercer la protección ante y contra otro Estado si constituye una traducción a términos jurídicos de la vinculación del individuo al Estado que lo ha hecho su nacional. Además, la nacionalidad produce sus más inmediatos, sus más extendidos y, para la mayoría de personas, sus únicos efectos dentro del orden jurídico del Estado que la ha otorgado. La nacionalidad sirve, ante todo, para determinar que la persona a quien se le confiere goza de los derechos y está sujeta a las obligaciones que la legislación de un Estado concede e impone a sus nacionales. Esto está implícitamente contenido en la noción más amplia de que la nacionalidad pertenece a la jurisdicción nacional del Estado"23.

Es por ello por lo que, si el Estado del que deriva la nacionalidad de una persona desaparece, también se extinguen el vínculo con sus ciudadanos y las obligaciones y la capacidad de protección. Por tanto, el resultado lógico sería el de la pérdida de nacionalidad y la ostentación de la categoría de apátrida, lo que conllevaría que esas personas no fueran reconocidas por ningún Estado como sujetos de derechos y obligaciones. Sin embargo, el progresivo desarrollo de los estándares de derechos humanos ha ido quebrando los fundamentos de la noción de que la nacionalidad es el derecho a tener derechos, en favor de un concepto de humanidad o dignidad humana como la base para el disfrute de los derechos ${ }^{24}$, lo que no supone un obstáculo para que los Estados continúen tratando de forma muy diferente a sus nacionales y a los no nacionales que se encuentran bajo su jurisdicción.

\footnotetext{
${ }^{23}$ Sentencia de la Corte Internacional de Justicia en el caso Nottebohm (Principado de Liechtenstein contra Guatemala), de 6 de abril de 1955, pp. 20-23.

${ }^{24}$ VAN WAAS, "Nationality Matters...", cit., p. 395, y voto razonado del juez Cançado Trindade en la Sentencia de la Corte Interamericana de Derechos Humanos (Caso Yean y Bosico contra República Dominicana), de 8 de septiembre de 2005, p. 2, disponible en: http://www.corteidh.or.cr/CF/Jurisprudencia2/busqueda_casos_contenciosos.cfm?lang=es.
} 
Por tanto, por una parte, se puede afirmar que la población afectada que se trasladaría a ese nuevo territorio seguiría conservando su nacionalidad en tanto en cuanto el Estado mantuviera su condición de tal, pero, incluso así, al desplazarse al territorio de otro Estado esas personas serían, de facto, apátridas. Y, por otra, en el momento en que se entendiera que el Estado desaparece (ya sea porque queda sumergido por las aguas del océano, ya sea porque deviene inhabitable en toda su extensión), la población desplazada devendría apátrida también de iure. En ambos casos la población experimentaría una importante pérdida de derechos. Tan precaria se considera la situación jurídica de los apátridas que incluso se han equiparado a una res nullius ${ }^{25}$.

Volviendo a las soluciones disponibles para los Estados insulares de baja altitud, si lo que se pretende es "trasladar" un Estado de un territorio inundado a otro situado en tierra firme conservando todos sus atributos, se debería optar por la cesión de parte del territorio de un Estado a otro. Sin embargo, si bien esta alternativa es posible, se trata de un escenario harto improbable. En este caso, el tratado bilateral estipulado entre ambos Estados debería recoger la cesión de soberanía al Gobierno del Estado "cesionario" para que este pudiese actuar con plenos poderes sobre el territorio cedido y su población. De esta forma, la población desplazada a esa zona conservaría su nacionalidad.

Otra de las alternativas para el Estado afectado por la pérdida total de su territorio pasaría por unirse a otro, lo que daría lugar probablemente a una absorción o incorporación por la cual el Estado "sin territorio" desaparecería para integrarse en el Estado receptor, que permanecería. En este escenario no habría ningún tipo de modificación territorial y, por lo que se refiere a la población del Estado que desaparece (o se incorpora), pasaría a tener la nacionalidad del Estado receptor, por lo que también en este caso el problema de la apatridia quedaría resuelto.

Visto el plano teórico, pasamos ahora al práctico, donde encontramos una serie de experiencias relativas a las posibilidades de actuación de los Estados más afectados por este fenómeno. Se puede tomar como ejemplo Tuvalu, cuyos atolones tienen una altitud promedio de menos de dos metros sobre el nivel del mar, y que puede ser el

${ }^{25}$ WEIS, P., Nationality and statelessness in international law, Ed. Stevens \& Sons Limited, Londres, 1956, p. 166. 
primer país en desaparecer a causa del cambio climático ${ }^{26}$. Consciente del peligro al que se enfrenta, este país ha sido pionero a la hora de buscar soluciones. Tras un proyecto fallido de comprar tierras en Texas en la década de 1980 y trasladar allí a su población ${ }^{27}$, intentó que tanto Australia como Nueva Zelanda aceptasen acoger un número anual de tuvaluanos en su territorio. Mientras que Australia rechazó la propuesta, Nueva Zelanda permite, desde 2002, la entrada anual de setenta y cinco tuvaluanos con edades comprendidas entre los 18 y los 45 años, bajo un esquema de migración laboral que también se aplica con respecto a Kiribati, Tonga y Fiyi, si bien el número de ciudadanos que se pueden beneficiar de esta medida varía dependiendo de su país de origen ${ }^{28}$.

Recientemente, en octubre de 2017, Nueva Zelanda dio un paso hacia el reconocimiento de los efectos del cambio climático como un motivo válido para buscar residencia en el país, quizás debido a los casos que abordan esta cuestión en los tribunales neozelandeses. Al menos, así lo anunció James Shaw, ministro para el Cambio Climático, al informar que el Gobierno neozelandés se estaba planteando crear un visado humanitario especial para las personas procedentes de las islas del Pacífico que se vean obligadas a abandonar sus hogares a causa del incremento del nivel del mar ${ }^{29}$. De esta forma, Nueva Zelanda prefiere optar por la adopción de medidas preventivas, adelantándose a la potencial situación de emergencia que se podría producir ante una avalancha de migrantes climáticos hacia sus fronteras.

Por su parte, el Gobierno australiano ha asegurado que invertirá 226 millones de dólares durante cuatro años para ayudar a las islas del Pacífico a combatir las consecuencias del cambio climático, pero ha señalado que no contempla desarrollar ningún plan migratorio similar al que propone Nueva Zelanda. Por el contrario, entiende que la mejor respuesta es la reubicación y la adaptación internas, tal y como indicaba un portavoz del ejecutivo australiano ${ }^{30}$.

\footnotetext{
26 https://www.efe.com/efe/america/sociedad/tuvalu-amenazado-con-desaparecer-pide-a-trumpresponsabilidad-el-clima/20000013-3264183

27 https://www.nytimes.com/1981/08/16/world/us-deal-embroils-tiny-island-nation.html

$28 \mathrm{https}: / /$ www.immigration.govt.nz/new-zealand-visas/apply-for-a-visa/about-visa/pacific-accesscategory-resident-visa

29 https://www.reuters.com/article/us-newzealand-climatechange-visa/new-zealand-considers-visa-forclimate-refugees-from-pacific-islands-idUSKBN1DH1JB

$30 \mathrm{http}: / / \mathrm{www}$.europapress.es/internacional/noticia-nueva-zelanda-planea-crear-visado-especialrefugiados-cambio-climatico-20171117162424.html
} 
Por lo que se refiere a la República de Kiribati, un país con una altura promedio de dos metros, sabemos que ha comprado dos mil hectáreas de tierra en la isla Vanua Levu, situada en Fiyi, adonde planea trasladar a sus cien mil habitantes en caso de que su Estado se vuelva inhabitable ${ }^{31}$. El presidente de Kiribati, Anote Tong, declaraba lo siguiente: "We would hope not to put everyone on [this] one piece of land, but if it became absolutely necessary, yes, we could do it"32. El problema es que Fiyi también debe lidiar con los efectos del cambio climático en su propio territorio, ya que desde 2012 ha tenido que reubicar a la población de algunas localidades costeras afectadas por las inundaciones en terrenos más altos en el interior ${ }^{33}$.

Por último, se pueden mencionar también las Islas Marshall, la mayoría de las cuales se encuentran a algo menos de dos metros por encima del nivel del mar ${ }^{34}$, a lo que se viene a sumar el hecho de que algunas de ellas tengan poco más de un kilómetro y medio de ancho. En el caso de este país, sus habitantes pueden emigrar libremente a Estados Unidos gracias a un acuerdo de libre asociación establecido entre los Gobiernos de ambos Estados ${ }^{35}$ cuya segunda enmienda estará en vigor hasta $2023^{36}$, aunque no existe la certeza de que se vaya a prolongar.

\section{La protección jurídica que reciben los migrantes por causas medioambientales y la problemática existente}

Pese a que los movimientos poblacionales provocados por cambios o circunstancias adversos, ya sean guerras o contiendas, ya sean de índole medioambiental, han sido siempre una estrategia habitual de adaptación humana, hoy en día debemos tener presentes las leyes estatales sobre la migración, que limitan la entrada de extranjeros en los Estados. Estos únicamente están obligados a dar protección a las personas

\footnotetext{
${ }^{31}$ http://www.climate.gov.ki/2014/05/30/kiribati-buys-a-piece-of-fiji/

$32 \mathrm{https}: / / w w w . t h e g u a r d i a n . c o m / e n v i r o n m e n t / 2014 / j u l / 01 /$ kiribati-climate-change-fiji-vanua-levu

${ }^{33} \mathrm{http}: / /$ newsroom.unfccc.int/es/cop23-bonn/asi-impacta-el-cambio-climatico-a-fiji/

34 "Most of the 1,000 or so Marshall Islands, spread out over 29 narrow coral atolls in the South Pacific, are less than six feet above sea level" (https://www.nytimes.com/interactive/2015/12/02/world/TheMarshall-Islands-Are-Disappearing.html).

${ }^{35}$ Nos estamos refiriendo al Compact of Free Association Act de 1986, en cuyo artículo IV, sección 141, se contempla la cuestión de la inmigración. Dicho texto legal se puede consultar en: https://www.doi.gov/oia/about/compact.

$36 \mathrm{http}: / /$ www.exteriores.gob.es/Documents/FichasPais/ISLASMARSHALL_FICHA\%20PAIS.pdf
} 
que cumplen las exigencias de determinados tratados internacionales de los que los Estados son parte, como es el caso de los refugiados o de los apátridas ${ }^{37}$.

Es por ello por lo que determinar quién es un refugiado y quién es estrictamente un migrante deviene fundamental, ya que implica que esa persona tiene un derecho legalmente establecido a recibir protección por parte del Estado de destino. A este fin, es importante distinguir entre algunos términos que a menudo se confunden, pues son difíciles de delimitar o de diferenciar con claridad.

El primero de ellos es el de migrante, un término muy complejo y difícil de definir, pues admite numerosas tipologías que no son excluyentes. De acuerdo con el concepto de migrante que maneja la Organización Internacional para las Migraciones ${ }^{38}$, se ha venido entendiendo que son personas que cambian su lugar de residencia habitual de forma voluntaria y por cuestiones económicas, principalmente. Sin embargo, no es difícil encontrar migrantes que se ven obligados a abandonar su lugar de residencia de forma forzosa, por lo que la migración, en términos generales, puede ser considerada como todo movimiento de personas que se desplazan hacia otro país o región desde su lugar de origen ${ }^{39} \sin$ tener en cuenta la razón de ese desplazamiento. Dicha consideración sobre el motivo del desplazamiento sí es importante, sin embargo, con vistas a la aplicación de un régimen legal u otro a la persona migrante.

Podemos distinguir multitud de clases o tipos de migrantes en función de determinadas variables. Por ejemplo, según el alcance de su movilidad, tendríamos migrantes internos (interprovinciales, interregionales) y migrantes internacionales; dependiendo de la duración de ese desplazamiento, hablaríamos de migraciones temporales, circulares ("refugiados en órbita") ${ }^{40} 0$ definitivas; si tenemos en cuenta la condición legal de los migrantes, distinguiríamos entre migrantes regulares y

\footnotetext{
${ }^{37}$ MCADAM, "El desplazamiento...", cit., p. 1

38 "Este término abarca usualmente todos los casos en los que la decisión de migrar es tomada libremente por la persona concernida por 'razones de conveniencia personal' y sin intervención de factores externos que le obliguen a ello. Así, este término se aplica a las personas y a sus familiares que van a otro país o región con miras a mejorar sus condiciones sociales y materiales y sus perspectivas y las de sus familias", Glosario sobre migración, OIM, 2006.

${ }^{39}$ ATAPATTU, S., "Climate change: disappearing states, migration, and challenges for international law", Washington Journal of Environmental Law \& Policy, 1, 2014, p. 23.

40 En palabras de Mariño Menéndez, los refugiados en órbita son "personas no reconocidas definitivamente como 'refugiados' en sentido propio por ningún Estado [...] porque no hay acuerdo entre los Estados con los que el 'buscador de refugio' haya tomado contacto respecto a cuál de ellos debe otorgar su protección a la persona en cuestión". MARIÑO MENÉNDEZ, F.M., "EI concepto de refugiado en un contexto de derecho internacional general”, REDI, vol. 35, núm. 2, 1983, p. 345.
} 
migrantes irregulares; en función de la modalidad migratoria, hablaríamos de migraciones individuales, familiares o grupales; y si reparamos en la voluntariedad del desplazamiento, distinguiríamos entre migraciones voluntarias o forzadas.

La clasificación que a nosotros nos interesa en este momento es la relativa a la causa que motiva la migración, pudiendo distinguirse entre causas económicas, familiares, medioambientales, políticas, religiosas, étnicas, sexuales, bélicas... El problema que surge aquí es que la migración es un fenómeno multicausal en el que intervienen también factores sociales, económicos, políticos y demográficos. Por tanto, no podemos afirmar que el cambio climático sea el factor clave de los desplazamientos forzosos, pues es casi imposible aislar ese factor del resto ${ }^{41}$. Por ejemplo, un conflicto armado, una situación de subdesarrollo, el fuerte crecimiento demográfico, la inestabilidad política o una mala planificación urbana debilitan la resiliencia de las personas afectadas y, al mismo tiempo, agravan el impacto del cambio climático y la degradación del medio ambiente ${ }^{42}$.

Esto significa que, en cuanto a la relación entre los movimientos migratorios y el cambio climático, se debe abandonar la idea de causa-efecto, pues, si bien es cierto que el cambio climático tiene efectos reales sobre la migración, estos se producen de forma más compleja.

Quizá la mejor definición de migrante por motivos medioambientales — por ser una definición muy amplia que pretende reflejar la complejidad de la cuestión al incluir movimientos voluntarios, forzados, internos, internacionales, de gradación lenta y de gradación extrema- sea la de la Organización Internacional para las Migraciones, que entiende que "los migrantes por motivos ambientales son personas o grupos de personas que, principalmente en razón de un cambio repentino o progresivo en el medio ambiente, que incide adversamente en sus vidas o en las condiciones de vida, se ven obligados a abandonar el lugar habitual de su residencia, u optan por hacerlo,

\footnotetext{
41 DUN, O. y GEMENNE, F., "Definir la migración por motivos medioambientales", Revista Migraciones Forzadas, núm. 31, 2008, pp. 10-11; o también SOLÀ PARDELL, O., "Desplazados medioambientales. Una nueva realidad", Cuadernos Deusto de Derechos Humanos, núm. 66, 2012, p. 38.

42 Agenda para la protección de las personas desplazadas a través de fronteras en el contexto de desastres naturales, p. 3, disponible en: https://disasterdisplacement.org/wpcontent/uploads/2015/03/02062016_Protection_Agenda_Final_SP.pdf.
} 
ya sea temporalmente o con carácter permanente, y que se desplazan dentro del país o al extranjero" (OIM, 2011:33).

Los movimientos de personas a causa del deterioro del entorno de los pequeños Estados insulares bajos que analizamos en estas líneas se pueden subsumir perfectamente en esta definición, pues, aunque la degradación viene provocada por la mano del hombre, no dejan de ser cambios en el medio los que dan lugar a la emigración.

Asimismo, las personas en estas circunstancias pueden entenderse también como un subtipo de migrante medioambiental, que venimos denominando migrante climático y cuya nota característica sería que esas modificaciones en el entorno estarían relacionadas con el cambio climático antropogénico. De ese modo, la subida del nivel del mar a causa del cambio climático (circunstancia diferenciadora de este término) provocaría la inhabitabilidad progresiva de un Estado (cambio progresivo en el medio ambiente) antes de hacerlo desaparecer bajo las aguas del océano, incidiendo adversamente en las condiciones de vida de sus habitantes, que se verían obligados a abandonar esa zona (mediante desplazamiento interno o internacional).

Habiendo abordado ya el término migrante, resta hacer una referencia a los desplazados y los refugiados, que son tipos particulares de migrantes. Ambos son movimientos forzados a causa de la violencia y se diferencian por el ámbito geográfico de movilidad: si el desplazamiento es interno, dentro de las fronteras de sus Estados, hablamos de desplazados, mientras que, si es un movimiento que traspasa fronteras, hablamos de refugiados.

La importancia de englobar a estas personas en una u otra categoría reside en el hecho de que les corresponderán regímenes jurídicos diferentes, de los que surgirán determinados derechos y obligaciones, como ya venimos afirmando.

Hecha esta distinción, la pregunta obligada que debemos responder es si las personas que migran de forma forzosa a causa de los efectos del cambio climático entrarían dentro de la categoría de refugiado. De no ser así, estos individuos corren el riesgo de ser considerados inmigrantes irregulares en el país al que se hayan desplazado, encontrándose en una situación de abandono y desprovistos de protección legal. 
Desde el punto de vista jurídico y con el marco del que disponemos para regular la figura del refugiado, que es la Convención de Ginebra de 1951, con su Protocolo de 1967, un migrante por causas medioambientales no sería un refugiado, pues, para atribuirle tal condición, debería reunir alguno de los motivos establecidos en el artículo 1.2 de dicha Convención ${ }^{43}$, es decir, ser perseguido por uno de los siguientes motivos: raza, religión, nacionalidad, grupo social u opiniones políticas.

Se ha planteado también la posibilidad de que los migrantes medioambientales puedan acogerse a la protección de 1951 en el caso de que sea su propio Gobierno el que intencionadamente contamine sus cultivos o las fuentes de agua de las que aquellos se abastecen o, en definitiva, haga inhabitable el entorno de una concreta comunidad $^{44}$. En estas circunstancias, el elemento persecutorio existiría y estaría ligado a una de las causas recogidas en la Convención de Ginebra. Es por ello por lo que la situación de estas personas se redirigiría a la categoría de refugiado, pero no por el elemento medioambiental presente, sino por la persecución que subyace a esas actuaciones que habría llevado a cabo el Gobierno y que, insistimos, debería tener como causa la raza, la religión, la nacionalidad, el grupo social o las opiniones políticas de los afectados.

\section{POSIBLES SOLUCIONES}

Tras haber analizado la complejidad y la situación de desamparo en la que se encuentran aquellos que sufren en primera persona los efectos del cambio climático en forma de subida del nivel del mar, el siguiente paso será exponer la variedad de soluciones que se pueden plantear para tratar de colmar ese vacío legal.

Para ello, procedemos a realizar una clasificación entre esas posibles respuestas, distinguiendo entre aquellas que se basan en instrumentos existentes y las que pasarían por la creación de nuevos instrumentos.

\footnotetext{
${ }^{43}$ Este artículo establece lo siguiente: "El término refugiado se aplicará a toda persona que debido a fundados temores de ser perseguida por motivos de raza, religión, nacionalidad, pertenencia a determinado grupo social u opiniones políticas, se encuentre fuera del país de su nacionalidad y no pueda o, a causa de dichos temores, no quiera acogerse a la protección de tal país; o que, careciendo de nacionalidad y hallándose, a consecuencia de tales acontecimientos, fuera del país donde antes tuviera su residencia habitual, no pueda o, a causa de dichos temores, no quiera regresar a él".

44 STAVROPOULOU, M., "¿Un mar de definiciones?, Cambio climático y desplazamiento", Revista Migraciones Forzadas, núm. 31, 2008, p. 12.
} 


\section{Instrumentos existentes}

Si partimos de los mecanismos de los que ya disponemos, son varias las alternativas con las que podemos trabajar.

En primer lugar, cabría señalar que, pese al debate generado inicialmente y a los intentos de que la definición de refugiado de la Convención de Ginebra se aplicara también a los migrantes por causas medioambientales ${ }^{45}$, lo cierto es que dicha equiparación no puede darse en términos jurídicos. Sin embargo, la expresión "refugiado medioambiental" continúa en uso, principalmente por parte de movimientos ciudadanos así como a nivel político, en un intento de dotarlo de mayor fuerza para generar un mayor impacto en la opinión pública y eliminar la connotación peyorativa que parece acompañar al término (in)migrante. Durante los últimos años, sin embargo, a causa de la guerra en Siria y la llamada "crisis de los refugiados"46, este término también ha absorbido ese tinte negativo. $Y$ no solo eso, sino que algunos de los pequeños Estados insulares incluso han expresado que no quieren ser considerados refugiados precisamente por la carga despectiva que acompaña al término ${ }^{47}$, ya que la situación de desamparo e indefensión sin perspectivas de futuro de los refugiados no se corresponde con su situación particular y con el fuerte orgullo pacífico ${ }^{48}$.

Dicho esto, y dejando a un lado la aplicación extensiva del concepto de refugiado de la Convención a los migrantes climáticos al haberse llegado a la conclusión de que no es algo factible, una posible solución que se ha venido planteando sería modificar la regulación de Ginebra y su definición de refugiado, ampliándola para dar cabida no solo a las personas perseguidas por su raza, religión, nacionalidad, grupo social u

\footnotetext{
45 Vid., en este sentido, RIVILLO TORRES, J., "Refugiados climáticos y Territorios Frontera", Redes.com, núm. 13, 2016, pp. 167-196.

${ }^{46}$ Vid., por ejemplo, GONZÁLEZ ENRÍQUEZ, C., "La crisis de los refugiados y la respuesta europea", Real Instituto Elcano, 2015; https://www.eldiario.es/theguardian/mitos-crisisrefugiados_0_782522278.html; o https://cnnespanol.cnn.com/video/crisis-refugiados-amenaza-unioneuropea-transformacion-confirmacion-politica-continente-acuerdo-control-flujo-controversia-brexitadriano-bosoni-entrevista-rodriguez-dinero/

47 http://insidestory.org.au/we-arent-refugees/; http://fijisun.com.fj/2016/12/13/people-displaced-byclimate-change-not-refugees-tuvalu-pm/; $\quad$ https://www.theguardian.com/vitalsigns/2014/sep/18/refugee-camps-climate-change-victims-migration-pacific-islands

48 MCADAM, J., "Refusing 'Refuge' in the Pacific: (de)constructing climate-induced displacement in international law", 2010, p. 12, disponible https://www.researchgate.net/publication/228196628_Refusing_\%27Refuge\%27_in_the_Pacific_DeC onstructing_Climate-Induced_Displacement_in_International_Law.
} 
opiniones políticas, sino también a quienes huyen de su hogar por circunstancias medioambientales ${ }^{49}$.

De optar por esta vía, las ventajas que obtendríamos serían claras. Por una parte, se estaría otorgando una elevada seguridad jurídica a estas personas y, por otra, les estaríamos dotando de los mismos derechos que la Convención contempla para los refugiados.

Por el contrario, el emprender la negociación de esta modificación podría dar lugar a una debilitación de un sistema ${ }^{50}$ que, pese a sus deficiencias, ha venido funcionando de forma aceptable. Asimismo, el sistema de 1951 también presenta limitaciones para abarcar esta nueva realidad, ya que no fue pensado para la complejidad que presentan las migraciones climáticas, sino que se fundamenta en un objetivo político específico que nació de la necesidad de gestionar el problema de las masas europeas de emigrantes tras la Segunda Guerra Mundial. Por lo tanto, no puede sorprendernos el hecho de que no se tuvieran en cuenta los factores medioambientales a la hora de elaborar este instrumento. Por último, pero no por ello menos importante, sino quizás todo lo contrario, no debemos perder de vista la resistencia que muestran los Estados para debatir y tratar la cuestión dentro del sistema de refugio de la Convención.

Otra vía de actuación que se podría plantear sería integrar el debate y su posible solución en el contexto de la Convención Marco de las Naciones Unidas sobre el Cambio Climático (CMNUCC) aprovechando el éxito, en términos de rapidez y número de ratificaciones, que ha alcanzado el Acuerdo de París. Esa gran acogida del instrumento de la COP-21 puso de manifiesto la preocupación compartida por el medio ambiente y la necesidad de actuar en aras de combatir el cambio climático, reconociéndose mundialmente su existencia, su origen antropogénico, la amenaza que supone y el vínculo entre migración, derechos humanos y cambio climático ${ }^{51}$.

49 En apoyo a esta propuesta encontramos autores como KOLMANNSKOG, V., "Climates of displacement", Nordic Journal of Human Rights, vol. 26, 2008, pp. 302-320; o BORRÁS PENTINAT, S., "Refugiados ambientales: el nuevo desafío del Derecho internacional del medio ambiente", Revista de Derecho, vol. 19, núm. 2, 2006, pp. 85-108.

${ }^{50}$ En este sentido se pronunciaba el propio António Guterres: "El ACNUR considera que con cualquier iniciativa para modificar esta definición se correría el peligro de una renegociación de la Convención de 1951, que en el entorno actual puede dar lugar a una reducción de las normas de protección del refugiado e incluso socavar todo el régimen de protección internacional de refugiado". GUTERRES, A., "Cambio climático, desastres naturales y desplazamiento humano: la perspectiva del ACNUR", documento de 23 de octubre de 2008, disponible en: http://www.unhcr.org/497891022.pdf.

51 Esta cuestión se debatió en la etapa de negociación del Acuerdo y, en un primer momento, se pensó en incluirla dentro del articulado del texto; sin embargo, finalmente se decidió "relegarla" a su 
Además, la Convención proporciona un marco jurídico e institucional bien establecido que se podría aprovechar para este proyecto.

Sin embargo, este sistema tampoco está exento de limitaciones. Por ejemplo, el hecho de que haya sido pensado principalmente para establecer medidas de mitigación de emisión de gases de efecto invernadero puede suponer una desventaja. O también el hecho más importante de que esta Convención Marco se basa en establecer relaciones entre los Estados, no entre los Estados y los individuos. Finalmente, otra de las desventajas de optar por esta alternativa sería que quedaría limitada a los desplazamientos provocados por el cambio climático, cuyo nexo causal, como ya hemos apuntado, es mucho más complejo que el de otras migraciones.

Descendiendo un escalón en el nivel de protección podríamos, simplemente, quedarnos en el ámbito del soft law y los principios que tenemos, aunando esfuerzos para desarrollarlos y conseguir, con el paso del tiempo, su plasmación en tratados o acuerdos de carácter vinculante. En este sentido tenemos el exitoso ejemplo de la Iniciativa Nansen, impulsada en el año 2012 por Noruega y Suiza como un proceso de consulta internacional que pudiese generar un amplio consenso entre Estados sensibilizados con la problemática de los desplazamientos por razones medioambientales y de cambio climático ${ }^{52}$. A través del establecimiento de una agenda operativa, su principal objetivo consiste en intentar evitar, en la medida de lo posible, que las personas afectadas por eventos climatológicos extremos o la degradación del medio se vean obligadas a abandonar su hogar y desplazarse dentro de su propio Estado o más allá de las fronteras de este. Este propósito podría materializarse mediante la adopción de las medidas de prevención necesarias en cada escenario concreto ${ }^{53}$; en el caso de que esta prevención no se hubiera podido realizar,

preámbulo, que recoge lo siguiente: "Reconociendo que el cambio climático es un problema de toda la humanidad y que, al adoptar medidas para hacerle frente, las Partes deberían respetar, promover y tener en cuenta sus respectivas obligaciones relativas a los derechos humanos, el derecho a la salud, los derechos de los pueblos indígenas, las comunidades locales, los migrantes, los niños, las personas con discapacidad y las personas en situaciones vulnerables y el derecho al desarrollo, así como la igualdad de género, el empoderamiento de la mujer y la equidad intergeneracional".

52 Vid. MARTÍN CUBEL, F., "El esfuerzo por el reconocimiento del refugiado medioambiental: la Iniciativa Nansen", IEEE, núm. 5, 2017, pp. 435-449.

53 Dichas medidas preventivas pueden ser muy variadas, pudiéndose implementar desde acciones de reducción del riesgo de desastres hasta medidas de adaptación o de reubicación planificada, pasando por la mejora de las infraestructuras. Vid. KÄLIN, W., "La Iniciativa Nansen: crear consenso sobre el desplazamiento en el contexto de los desastres", Revista de Migraciones Forzadas, núm. 49, 2015, pp. 5-7. 
la iniciativa plantea que los Estados otorguen una protección efectiva, determinada y consensuada a las personas afectadas por estos desastres medioambientales.

El punto fuerte a destacar de este planteamiento sería la mayor facilidad a la hora de negociar estos principios o recomendaciones y de lograr el apoyo de los Estados debido al carácter no vinculante de dichos principios. Asimismo, también sería posible que las negociaciones tuvieran en cuenta en mayor medida las necesidades regionales o nacionales, pudiéndose adaptar a ellas. Sin embargo, todo ello, aunque dota de agilidad al proceso, implica resignarse a establecer un nivel de protección bajo cuya efectividad dependerá del compromiso y la voluntad de los Estados.

Antes de pasar a la segunda categoría de instrumentos, debemos hacer mención en este apartado a un régimen de protección inamovible: el de los derechos humanos.

Independientemente de que no sean pocas las incertidumbres que rodean al tema que abordamos en este estudio, si de algo estamos seguros es de que las personas que se ven obligadas a abandonar su hogar a causa de la destrucción de su entorno no pueden ser despojadas de sus derechos humanos más fundamentales, inherentes a toda persona.

Por tanto, ese vacío aparente de protección internacional podría verse colmado por esta rama del derecho. Entre otras cosas, de esta forma se solventaría la cuestión terminológica jurídica ${ }^{54}$, pues, al margen de las circunstancias personales de cada individuo, ya sea nacional, apátrida, refugiado o migrante, o incluso si no encaja en ninguna de estas categorías, sería igualmente objeto de protección acudiendo al régimen de los derechos humanos, derivado tanto de los tratados internacionales ratificados por los Estados como de la costumbre internacional.

Así, además de los derechos humanos absolutos, como sería el derecho a la vida, sin el cual el resto de derechos no tendrían razón de ser, estas personas gozarían de otra serie de derechos que, bajo el principio de no discriminación, no admitirían, por el mero hecho de que una persona fuera extranjera, un tratamiento diferenciado del que

\footnotetext{
${ }^{54}$ ESPÓSITO, C. y TORRES CAMPRUBÍ, A., "Cambio climático y derechos humanos: El desafío de los 'nuevos refugiados"', Revista de Derecho Ambiental de la Universidad de Palermo, núm. 1, 2012, p. 20.
} 
recibiría un nacional ${ }^{55}$. Esos derechos serían, entre otros, la libertad de pensamiento, conciencia y religión, los derechos garantizados en un juicio o la prohibición de ser sometido a tortura o tratos crueles, inhumanos o degradantes.

Además, y dado que el cambio climático pone en peligro el disfrute pleno de derechos tales como el derecho a la vida, la salud, el agua, la alimentación, la vivienda, el desarrollo o la autodeterminación ${ }^{56}$, podría plantearse la aplicación del mecanismo de la protección subsidiaria para amparar a estas personas. Este tipo de protección, en palabras de McAdam, la otorgan los Estados a partir de una necesidad de protección internacional fuera del marco de la Convención de Ginebra y puede basarse en un tratado de derechos humanos o en principios humanitarios más generales, como otorgar asistencia a personas que huyen de situaciones de violencia generalizada ${ }^{57}$.

En el ámbito de la UE, por ejemplo, disponemos de la Directiva de Protección Temporal ${ }^{58}$, que, sin recoger explícitamente en su texto los términos migrante o desplazado climático o medioambiental, en su artículo 2 abre la posibilidad a que las masas de migrantes de terceros países o apátridas que (1) hayan huido de zonas de conflicto armado o violencia permanente, o (2) hayan estado o estén en peligro grave de verse expuestos a una violación sistemática o generalizada de los derechos humanos, puedan ser objeto de este tipo de protección.

Por consiguiente, tanto si entendemos que estas personas huyen de su país de origen porque la situación medioambiental (una catástrofe repentina o una situación resultado de un deterioro gradual que alcanza niveles de inhabitabilidad o cercanos a ella) está lesionando determinados derechos (vida, seguridad, agua, salud, alimentación...), como si entendemos que es el Gobierno el que provoca esa violación al no ser capaz de asegurarlos ${ }^{59}$, cabría considerar que dichas circunstancias entran

\footnotetext{
55 ALEINIKOFF, A., "Un análisis sobre las Normas Jurídicas Internacionales y Migración", Diálogo Internacional sobre la Migración, núm. 3, OIM, Ginebra, 2002, disponible en: http://publications.iom.int/system/files/pdf/idm3_sp.pdf.

56 KNOX, J. H., "Cambio climático y derechos humanos", 2016, disponible en: https://observatoriosociallacaixa.org/-/cambio-climatico-y-derechos-humanos.

${ }^{57}$ MCADAM, J., Complementary protection in international refugee law, Oxford University Press, Oxford, 2007, p. 21.

58 Directiva 2001/55 del Consejo, de 7 de agosto de 2001, relativa a las normas mínimas para la concesión de protección temporal en caso de afluencia masiva de personas desplazadas y a medidas de fomento de un esfuerzo equitativo entre los Estados miembros para acoger a dichas personas y asumir las consecuencias de su acogida.

59 KOLMANNSKOG, V., y MYRSTAD, F., "Environmental Displacement in European Asylum Law", European Journal of Migration and Law, vol. 11, 2009, p. 320. Pese a estas aproximaciones doctrinales,
} 
dentro de las situaciones contempladas en el segundo de los apartados, lo que daría lugar a ese tipo de protección en concreto.

Pero incluso haciendo esta interpretación extensiva de lo expuesto en la Directiva (y que implicaría una voluntad política que hoy en día no se da), la protección tendría un carácter, como su propio nombre indica, temporal, ofreciendo un remedio pasajero, provisional, y no una solución definitiva. Lo deseable sería un remedio permanente, ya que los migrantes, a causa de la subida del nivel del mar, no podrían regresar a su hogar cuando en este se llegase a un tipping point o punto de no retorno, que se alcanzaría cuando el territorio se hubiera convertido en inhabitable en su conjunto o en la práctica totalidad de su extensión.

Más complicado aún resultaría, por otro lado, intentar argumentar que los migrantes climáticos entrarían dentro del ámbito de aplicación de la Directiva de Cualificación ${ }^{60}$, por cuanto su artículo 2 f) estipula que el solicitante que no reúna las condiciones para obtener el estatuto de refugiado puede recibir protección subsidiaria si se considera que se enfrenta a un riesgo real de sufrir un daño grave, entendiéndose este, ex artículo 15, como (1) la condena a pena de muerte o su ejecución, (2) la tortura o las penas o tratos inhumanos o degradantes en su país de origen, o (3) las amenazas graves e individuales contra la vida o la integridad física de un civil motivadas por una violencia indiscriminada en situaciones de conflicto armado internacional o interno.

A todo ello hay que sumar lo que ocurre en la práctica, donde encontramos una resistencia importante a declarar a un migrante climático como beneficiario de ayuda por la vía de la protección complementaria. Esto es lo que viene sucediendo en tribunales que tratan sobre estos casos desde hace algunos años, como ocurre en Nueva Zelanda. Pese a que los medios de comunicación hablasen en 2014, erróneamente, de que una decisión del Tribunal de Inmigración y Protección de Nueva

nos alineamos con el planteamiento de Espósito y Torres, ya que parece difícil aceptar que se hable de violación de derechos humanos por parte de un Estado cuando ni siquiera se encuentra en condiciones de ofrecer protección a sus ciudadanos y, menos aún, cuando los mayores responsables de los efectos del cambio climático son los Estados desarrollados (ESPÓSITO y TORRES CAMPRUBí, "Cambio climático y...", cit., p. 22).

60 Directiva 2011/95/UE del Parlamento Europeo y del Consejo, de 13 de diciembre de 2011, por la que se establecen normas relativas a los requisitos para el reconocimiento de nacionales de terceros países o apátridas como beneficiarios de protección internacional, a un estatuto uniforme para los refugiados o para las personas con derecho a protección subsidiaria y al contenido de la protección concedida. 
Zelanda ${ }^{61}$ había reconocido a los primeros "refugiados climáticos" del mundo62, la realidad es otra bien distinta. Si bien el Tribunal concedió a la familia de Tuvalu visados de residencia permanentes para quedarse en el país, lo cierto es que no lo hizo sobre la base de los impactos que el cambio climático está teniendo en Tuvalu ni porque los miembros de dicha familia estuviesen legitimados para recibir protección como refugiados o vía protección complementaria, sino que apreció otras circunstancias humanitarias, como fue el caso de los fuertes vínculos familiares que los unían con Nueva Zelanda ${ }^{63}$.

\section{Nuevos instrumentos}

La segunda categoría de respuestas que se nos plantea implicaría la elaboración y el desarrollo de nuevos instrumentos. Así, podemos abordar la posibilidad de elaborar un tratado internacional específico, ad hoc, sobre la cuestión de los migrantes climáticos. Este sería el caso de las propuestas de varias universidades, entre ellas la de Limoges, Harvard o Ámsterdam ${ }^{64}$.

La última propuesta define los refugiados climáticos como personas que tienen que abandonar sus hábitats, inmediatamente o en un futuro próximo, a causa de alteraciones repentinas o graduales en su medio natural relacionadas, al menos, con uno de los siguientes impactos del cambio climático: la subida del nivel del mar, los fenómenos meteorológicos extremos o las sequías y la escasez de agua.

Biermann y Boas ${ }^{65}$ abogan por la elaboración de un protocolo sobre reconocimiento, protección y reasentamiento de estas personas en el marco de la CMNUCC, que se podría establecer con el apoyo de los países que ya son parte de la Convención

\footnotetext{
${ }^{61}$ Decisión del Tribual de Inmigración y Protección de Nueva Zelanda, de 4 de junio de 2014, disponible en: http://www.nzlii.org/nz/cases/NZIPT/2014/501370.html.

62 Entre otros, https://www.smithsonianmag.com/smart-news/worlds-first-climate-change-refugeeswere-just-granted-residency-new-zealand-180952279/; https://grist.org/living/the-worlds-first-officialclimate-refugees-land-in-new-zealand/; https://inhabitat.com/worlds-first-real-climate-change-refugeesarrive-in-new-zealand/.

63 MCADAM, J., "No 'Climate Refugees' in New Zealand", 2014, disponible en: https://www.brookings.edu/blog/planetpolicy/2014/08/13/no-climate-refugees-in-new-zealand/.

64 LÓPEZ RAMÓN, F., "Los derechos de los emigrantes ecológicos", Cuadernos Manuel Giménez Abad, núm. extraordinario 6, 2017, pp. 13 y 14.

${ }^{65}$ BIERMANN, F. y BOAS, I., "Protecting climate refugees: the case for a global protocol", Environment Magazine, vol. 50, núm. 6, 2008, pp. 9-16.
} 
basándose en los principios ampliamente aceptados y aprovechando los avances científicos para definir los riesgos a los que se enfrentan estas personas.

Sus autores proponen que el acuerdo opere bajo cinco principios, a saber: (1) su objetivo debe descansar en un reasentamiento y una reintegración planeados y voluntarios, evitando ofrecer una mera respuesta de emergencia y ayuda en casos de catástrofe; (2) los "refugiados climáticos" deben ser considerados y tratados como inmigrantes permanentes que no pueden volver a su lugar de origen o residencia; (3) el régimen de estas personas debe diseñarse desde la constatación de que los migrantes son grupos de población que pueden ir desde una pequeña aldea a una nación entera, como sería el caso de los pequeños Estados insulares, por lo que se debe abandonar el enfoque de las necesidades individuales; (4) este régimen de protección buscará apoyar a Gobiernos, comunidades locales y organismos nacionales para que estén en condiciones de ofrecer protección a las personas en su territorio; y (5) esta protección de los "refugiados climáticos" debe verse como un problema y una responsabilidad globales, por lo que los países industrializados más ricos deben aportar financiación y apoyo para favorecer la protección y el reasentamiento de aquellos.

Además de estos principios rectores, los promotores de este instrumento concretan algunos extremos de este. Por ejemplo, consideran que el mecanismo de gobernanza más importante del acuerdo sería una lista de zonas bajo jurisdicción de los Estados miembros cuya población se encuentre amenazada o en situación de necesitar una reubicación a causa del cambio climático. Además, contemplan la creación de un comité ejecutivo que funcionaría bajo la autoridad de las partes y que determinaría la inclusión en la lista de las zonas propuestas por los Estados, así como las posibles medidas de apoyo.

Como cualquier medida a implementar, este sistema de protección precisaría de un fondo propio, independiente, bajo la autoridad de los Estados parte, que se podría nutrir de impuestos nuevos, como una tasa aplicada a los vuelos internacionales.

En 2009 se publicó una propuesta de Harvard, de la mano de Docherty y Giannini66, que recoge el concepto de "refugiado a causa del cambio climático" y lo define como

66 DOCHERTY, B. y GIANNINI, T., "Confronting a rising tide: a proposal for a Convention on climate change refugees", Harvard Environmental Law Review, vol. 33, núm. 2, 2009, pp. 349-405. 
un individuo que se ve forzado a huir de su hogar y a trasladarse, temporal o permanentemente, más allá de sus fronteras nacionales como resultado de alteraciones repentinas o graduales del medio ambiente probablemente provocadas por el cambio climático antropogénico.

A diferencia de la propuesta holandesa, estos autores "exigen" que haya un cruce de fronteras para que se pueda calificar a una persona como refugiado a causa del cambio climático.

Otra diferencia con respecto al modelo anterior sería que los profesores de Harvard consideran que la cuestión de las migraciones relacionadas con el cambio climático es lo suficientemente novedosa y posee la suficiente entidad propia como para gozar de una regulación independiente, sin necesidad de articularla en forma de protocolo anejo a la Convención de Ginebra o a la CMNUCC. Así, los desafíos existentes se podrían abordar mejor, y las negociaciones se podrían plantear de una forma diferente a la habitual Estado-Estado, permitiendo la presencia de comunidades y de la sociedad civil.

Con este instrumento se pretende compartir la responsabilidad entre los Estados afectados por el cambio climático, aquellos receptores de los flujos migratorios y la comunidad internacional a la hora de asegurar que se protegen los derechos de estas personas y que reciben la ayuda humanitaria necesaria.

Como no podía ser de otra manera, esta propuesta también contempla la creación de un fondo para implementar las medidas del acuerdo, una agencia de coordinación y un equipo de científicos para tomar decisiones relativas a la interpretación del instrumento y al reparto de responsabilidades financieras.

El proyecto que mayor repercusión parece estar teniendo, al menos en el ámbito académico y doctrinal, es el de la universidad francesa de Limoges $^{67}$, que ya va por su cuarta versión ${ }^{68}$. Se trata de un texto bastante completo que, bajo el formato de un tratado con un preámbulo y un articulado dividido en capítulos, combina responsabilidad y protección. Su objetivo es garantizar los derechos de los desplazados ambientales, a los que define como personas físicas, familias, grupos y

$67 \mathrm{https}: / /$ cidce.org/deplaces-environnementaux-refugies-ecologiques-environmentally-displacedpersons/.

68 La cuarta versión es muy reciente, pues es de mayo de este mismo año. 
poblaciones que se enfrentan a un cambio repentino o gradual de su medio ambiente que inevitablemente pone en peligro sus condiciones de vida, obligándoles a abandonar, en una emergencia o a largo plazo, su lugar de residencia habitual.

La adopción de un nuevo acuerdo permitiría disponer de un instrumento actual y completo, ya que estaría adaptado a todas las necesidades y complejidades que presentan estos movimientos de población. También conllevaría la creación de una estructura institucional propia y adaptada a las necesidades que surgieran, además de la estructura financiera correspondiente. Por otro lado, entre los puntos débiles de estas iniciativas, cabe señalar la dificultad para evitar tanto la impasibilidad como la resistencia de la comunidad internacional a optar por alguno de estos instrumentos.

Y es que hay algo que debe tenerse presente en todo momento. Una cosa es que esta solución sea la más deseable y otra que se obvie el contexto internacional, que, por desgracia, no es favorable a la adopción de nuevos acuerdos obligatorios, al menos en esta materia. Por tanto, debemos ser conscientes de que se debe trabajar con la realidad existente y no con la que nos gustaría tener.

Continuando con los acuerdos jurídicamente vinculantes, dado que lograr la aprobación de uno por parte de toda la comunidad internacional es actualmente una utopía, optar por la elaboración de acuerdos regionales o bilaterales puede ser un objetivo más factible que podría extenderse con el tiempo.

En ese caso, no habría que partir de cero en las negociaciones, pues se podrían utilizar como base acuerdos existentes como el de EE. UU. y las Islas Marshall, que, si bien no fue diseñado para hacer frente al cambio climático y sus consecuencias para los desplazamientos humanos, puede servir de referente.

Si optamos por este tipo de acuerdos más restringidos, en principio encontraríamos una mayor facilidad para negociarlos y para alcanzar un consenso. Además, las respuestas estarían más adaptadas a las necesidades regionales, lo que es muy importante si tenemos en cuenta que, según las previsiones, los desplazamientos tendrán lugar sobre todo dentro del mismo país o de una misma región ${ }^{69}$.

69 http://acnur.es/noticias/notas-de-prensa/2669-preguntas-frecuentes-sobre-desplazamiento-porcambio-climatico-y-desastres-naturales 
Entre las desventajas de este tipo de acuerdos, nos encontraríamos, primero, con la posibilidad de que surgieran desequilibrios entre las distintas respuestas regionales $y$, segundo, con el resigo de que dichas respuestas quedaran restringidas únicamente a los países y regiones más afectados por estos movimientos poblacionales, que presumiblemente serán los que tomen la iniciativa a la hora de elaborar y tratar de aprobar dichos acuerdos.

\section{CONCLUSIONES}

Como hemos visto, el cambio climático está poniendo a prueba la capacidad de la comunidad internacional para dar respuesta tanto a los impactos que este fenómeno ya está causando como a aquellos que no ha causado todavía pero que, según indican numerosos estudios al respecto, sabemos que causará en un futuro más o menos cercano.

Sin embargo, pese a que los pequeños Estados insulares de baja altitud ya han dado la voz de alarma en incontables ocasiones, parece que el asunto no se considera como un problema apremiante ni global por el resto de Estados que no se verían afectados, o al menos no de forma significativa, por las consecuencias devastadoras de la subida del nivel del mar.

Y es que nada se puede hacer si no existe una voluntad política de abordar esta cuestión de forma directa y eficaz. De poco servirán los numerosos foros intergubernamentales relacionados con el cambio climático que se han creado en los últimos años si finalmente los Estados no adoptan ningún instrumento legalmente vinculante que les obligue a prestar atención, ayuda y protección a las personas que se ven obligadas a huir y abandonar su hogar por circunstancias que en gran medida son consecuencia de la actividad contaminante del ser humano.

Existen soluciones, propuestas, iniciativas, proyectos. Por tanto, no cabe excusarse en la complejidad del problema o en la falta de alternativas. La comunidad internacional debe tomar en serio el principio de las responsabilidades comunes pero diferenciadas ya no solo aportando su ayuda financiera o tecnológica a los países que más la necesitan, sino haciendo también todo lo posible por adoptar una solución que 
ponga remedio a las necesidades que afrontan los migrantes climáticos con vistas a preservar su dignidad y a hacer efectivos sus derechos.

\section{BIBLIOGRAFÍA}

ALEINIKOFF, A., "Un análisis sobre las Normas Jurídicas Internacionales y Migración", Diálogo Internacional sobre la Migración, núm. 3, OIM, Ginebra, 2002.

ATAPATTU, S., "Climate change: disappearing states, migration, and challenges for international law", Washington Journal of Environmental Law \& Policy, 1, 2014.

BIERMANN, F. y BOAS, I., "Protecting climate refugees: the case for a global protocol", Environment Magazine, vol. 50, núm. 6, 2008, pp. 9-16.

BORRÁS PENTINAT, S., "La migración ambiental: entre el abandono, el refugio y la protección internacional", PAPELES de relaciones ecosociales y cambio global, núm. 132, 2016, pp. 31-49.

BORRÁS PENTINAT, S., "Refugiados ambientales: el nuevo desafío del Derecho internacional del medio ambiente", Revista de Derecho, vol. 19, núm. 2, 2006, pp. 85108.

DECONTO, R. y POLLARD, D., "Contribution of Antarctica to past and future sealevel rise”, Nature, núm. 531, 2016, pp. 591-597.

DÍEZ DE VELASCO, M., Instituciones de derecho internacional público, Ed. Tecnos, Madrid, 2009, 17. ${ }^{\text {a }}$ ed.

DOCHERTY, B. y GIANNINI, T., "Confronting a rising tide: a proposal for a Convention on climate change refugees", Harvard Environmental Law Review, vol. 33, núm. 2, 2009, pp. 349-405.

DUN, O. y GEMENNE, F., "Definir la migración por motivos medioambientales", Revista Migraciones Forzadas, núm. 31, 2008, pp. 10-11.

ESPÓSITO, C. y TORRES CAMPRUBÍ, A., "Cambio climático y derechos humanos: El desafío de los 'nuevos refugiados'”, Revista de Derecho Ambiental de la Universidad de Palermo, núm. 1, 2012, pp. 7-32.

FRIPP, E., Nationality and statelessness in the international law of refugee status, Bloombsbury, Londres, 2016.

GONZÁLEZ ENRÍQUEZ, C., "La crisis de los refugiados y la respuesta europea”, Real Instituto Elcano, 2015. 
GUTERRES, A., "Cambio climático, desastres naturales y desplazamiento humano: la perspectiva del ACNUR", documento de 23 de octubre de 2008, disponible en: http://www.unhcr.org/497891022.pdf.

JAKSA, B. y SMITH, J., "África: de los principios voluntarios a las normas vinculantes", Revista Migraciones Forzadas, núm. especial, diciembre de 2008, pp. 18-19.

KÄLIN, W., "La Iniciativa Nansen: crear consenso sobre el desplazamiento en el contexto de los desastres", Revista de Migraciones Forzadas, núm. 49, 2015, pp. 5-7.

KNOX, J. H., "Cambio climático y derechos humanos", 2016, disponible en: https://observatoriosociallacaixa.org/-/cambio-climatico-y-derechos-humanos.

KOLMANNSKOG, V., "Climates of displacement", Nordic Journal of Human Rights, vol. 26, 2008, pp. 302-320.

KOLMANNSKOG, V. y MYRSTAD, F., "Environmental Displacement in European Asylum Law", European Journal of Migration and Law, vol. 11, 2009, pp. 313-326.

LÓPEZ RAMÓN, F., "Los derechos de los emigrantes ecológicos", Cuadernos Manuel Giménez Abad, núm. extraordinario 6, 2017, pp. 6-18.

MARIÑO MENÉNDEZ, F. M., "El concepto de refugiado en un contexto de derecho internacional general”, REDI, vol. 35, núm. 2, 1983, pp. 337-370.

MARTÍN CUBEL, F., "El esfuerzo por el reconocimiento del refugiado medioambiental: la Iniciativa Nansen", IEEE, núm. 5, 2017, pp. 435-449.

MCADAM, J., Complementary protection in international refugee law, Oxford University Press, Oxford, 2007.

MCADAM, J., "El desplazamiento provocado por el cambio climático y el derecho internacional", Evento paralelo al Diálogo del Alto Comisionado sobre los desafíos en materia de protección, 8 de diciembre de 2010, Palacio de las Naciones, Ginebra.

MCADAM, J., "No 'Climate Refugees' in New Zealand", 2014, disponible en: https://www.brookings.edu/blog/planetpolicy/2014/08/13/no-climate-refugees-in-newzealand/.

MCADAM, J., "Refusing 'Refuge' in the Pacific: (de)constructing climate-induced displacement in international law", 2010.

MENGEL, M., LEVERMANN, A., FRIELER, K., ROBINSON, A., MARZEION, B. y WINKELMANN, R., "Future sea level rise constrained by observations and long-term commitment", Proceedings of the National Academy of Sciences, vol. 113, núm. 10, 2016, pp. 2597-2602.

NEREM, R. S., BECKLEY, B. D., FASULLO, J. T., HAMLINGTON, B. D., MASTERS, D. y MITCHUM, G. T., "Climate-change-driven accelerated sea-level rise", Proceedings of the National Academy of Sciences, 2018. 
PARK, S., "El cambio climático y el riesgo de apatridia: la situación de los Estados insulares bajos", Política de Protección y Asesoría Legal Series de Investigación, PPLA/2011/04, 2011.

RIVILLO TORRES, J., "Refugiados climáticos y Territorios Frontera", Redes.com, núm. 13, 2016, pp. 167-196.

SOLÀ PARDELL, O., "Desplazados medioambientales. Una nueva realidad", Cuadernos Deusto de Derechos Humanos, núm. 66, 2012.

STAVROPOULOU, M., "¿Un mar de definiciones?, Cambio climático y desplazamiento", Revista Migraciones Forzadas, núm. 31, 2008, pp. 11-12.

VAN WAAS, L., Nationality Matters. Statelessness under international law, Intersentia, Amberes, 2008.

WEIS, P., Nationality and statelessness in international law, Ed. Stevens \& Sons Limited, Londres, 1956.

YASUNAGA KUMANO, M., "Los Pequeños Estados Insulares en Desarrollo y los desplazados climáticos", IEEE, núm. 4, 2016, pp. 497-511. 